\title{
Cultura Ambiental en estudiantes de educación superior, 2020
}

Environmental Culture in Higher Education Students, 2020

\author{
Mariela Alexi Díaz ${ }^{*}$ (ID \\ Universidad Técnica Estatal de Quevedo \\ Juan Pablo Urdánigo Zambrano ${ }^{2}$ \\ Universidad Técnica Estatal de Quevedo \\ Anahí Mercedes Gallardo ${ }^{3 *}$ \\ Universidad Técnica Estatal de Quevedo \\ Ronald Hitler Muñoz Casanova ${ }^{4}$ iD \\ Universidad Técnica Estatal de Quevedo
}

Fecha recepción: 15 de junio de 2020

Fecha aceptación: 16 de julio de 2020

(1) 2020 Universidad de Cordoba. Este es un artículo de acceso abierto distribuido bajo los términos de la licencia Creative Commons Attribution License, que permite el uso ilimitado, distribución y reproducción en cualquier medio, siempre que el autor original y la fuente se acreditan.

\footnotetext{
${ }^{1}$ Máster en Administración Ambiental, Universidad Técnica Estatal de Quevedo, Integrante del grupo de investigación Valoración y Calidad Ambiental /Facultad Ciencias Ambientales, Quevedo, Ecuadory mdiaz@uteq.edu.ec, 0000-0001-8944-5994 *

2 Máster en Gestión Ambiental, Universidad Técnica Estatal de Quevedo, Integrante del grupo de investigación Biología de la conservación /Facultad Ciencias Ambientales, Quevedo, Ecuadory jurdanigo@uteq.edu.ec,0000-0002-8972-0279

3 Magister en Salud de los Trabajadores, Universidad Técnica Estatal de Quevedo, Docente/Salud Ocupacional y Seguridad en el Trabajo/Facultad Ciencias de la Ingeniería, Quevedo, Ecuador, emartinez@uteq.edu.ec, https://orcid.org/0000-0002-6433-2173

${ }^{4}$ Ingeniero en Gestión Ambiental, Universidad Técnica Estatal de Quevedo, Integrante del grupo de investigación Biología de la conservación /Facultad Ciencias Ambientales, Quevedo, Ecuador y ronald.munoz@uteq.edu.ec,0000-0002-0517-0354
} 


\section{RESUMEN}

La formación de profesionales sin cultura ambiental; atenta al desarrollo sostenible de las sociedades. El nivel y calidad de educación, es un factor preponderante en la toma de decisiones. La evaluación del nivel de cultura ambiental en los estudiantes universitarios se basó en la encuesta modificada de Wisconsin; a 2366 estudiantes de una institución de educación superior, herramienta que analiza las actitudes, comportamientos y conocimientos con enfoque ambiental. Se utilizó el programa Statistical Package for the Social Sciences V22 para el análisis estadístico inferencial no paramétrico de U - Mann Whitney y Kusskal Wallis, que asigna un valor de 4 como favorable y 0 como menos favorable a cada pregunta. Los resultados obtenidos en cada aspecto evidencian para la actitud, una elevada $(2,96)$ preocupación por los riesgos para la salud ambiental; sin apoyo a las regulaciones de contaminación en una comunidad, al considerar que interfieren con el crecimiento industrial y desarrollo $(1,28)$. Apagar las luces y los electrodomésticos; cuando no se utilizan para ahorrar electricidad, es el comportamiento más aplicado por los estudiantes universitarios $(2,96)$. Sin embargo, no poseen interés en el envío de cartas al periódico sobre problemas ambientales $(1,14)$. El conocimiento más destacado en los universitarios, es la definición del hábitat $(2,65)$; siendo notoria la inconsciencia del impacto antropogénico en la elevada tasa de extinción de especies $(0,91)$. El Índice de Cultura Ambiental en los estudiantes promedió 5,1; definida como inaceptable. El análisis de clúster avala la escasa cultura ambiental, por la fuerte

\section{ABSTRACT}

La formación de profesionales sin cultura ambiental, es un atentado a la sostenibilidad en el desarrollo de las sociedades; debido que el nivel y calidad de educación, incide como factor preponderante en la toma de decisiones.

The research shows the level of environmental culture existing in university students. 2366 students from the various careers of a higher education institution were surveyed. For the evaluative phase, the modified Wisconsin survey was used, which analyzed the attitudes, behaviors and knowledge with an environmental focus in the students of the different academic units. The statistical program SPSS V22 was used; Descriptive statistics, non-parametric inferential statistics and multivariate statistics were applied. The average of environmental culture in the students was 5.1; which determined an unacceptable Environmental Culture Index. The incipient concern for environmental problems is shown in the poor correlation of the questions in the cluster analysis. The concern for environmental health risks and the belief of being able to contribute to the solution of environmental problems with actions, obtained the highest valuations in the total averages. Which shows that despite having superficial knowledge of the environmental reality and the importance of the actions they carry out, what they think does not take effect. The results show that teachers influence certain attitudes and behaviors of students, who must take advantage of the interaction space and academic activities to increase environmental culture through theoretical and practical motivational 
correlación entre el desinterés de comunicar los problemas ambientales a los periódicos y denunciar las violaciones ambientales a las autoridades. El incremento de la cultura ambiental, precisa del aprendizaje ambiental transversal en todas las profesiones; que incluyan acciones prácticas aplicadas a la realidad, para modelar las actitudes hacia el alcance de los Objetivos del Desarrollo Sostenible.

PALABRAS CLAVE: enviromental education,enviromental culture, university students, university sustainability, sustainable development

\section{INTRODUCCIÓN}

Los ejes sociales, económicos, políticos y culturales desestructuran a los ecosistemas naturales, superando su capacidad de resistencia, adaptación y poniendo en serio peligro el equilibrio dinámico. Se requiere de un cambio social con carácter revolucionario que a la vez necesita de un cambio educativo: no hay cambio social consciente sin una medición educativa (Alba Hidalgo, 2017). La educación ambiental se inscribe como la estrategia actual de cómo abordar la problemática ambiental, más allá de plantear solo respuestas eminentemente técnicas o tecnológicas, como aspectos claves del desarrollo sostenible en los ámbitos locales (Reyes Pineda \& Hérnandez, 2015). Un cambio cultural-ambiental es necesario y strategies. To make it; teachers must be constantly trained in the environmental area.

KEYWORDS: educación ambiental, cultura ambiental, estudiantes universitarios, sostenibilidad universitaria, desarrollo sostenible fundamental en los pensamientos, actitudes y aptitudes por parte de todos los profesionales y líderes sociales, así como de la población en general (Hidalgo D., 2017).

La cultura ambiental, además de ser un factor que determina la conducta del individuo con los semejantes, también lo hace con el entorno. Incluye el comportamiento a favor del ambiente; indicando que el conocimiento que los individuos adquieren de forma empírica y teórica, podría tener influencia en sus actitudes. Por lo tanto, el bajo conocimiento del entorno socioecológico es un factor importante en la modificación desmedida del ambiente (Vacio Fraga, 2017). Las diferencias culturales son cruciales al relacionar los valores culturales con los valores proambientales a la hora de generar 
propuestas que procuren la sostenibilidad. Las universidades son un nicho ideal; dado que los valores proambientales bien orientados se internalizan como obligaciones morales, haciendo más efectivas las campañas de modelación conductual en relación a la protección y cuidado ambiental (Chwialkowska et al., 2020)

La garantía del bienestar integral de las presentes y futuras generaciones; que incluye las dimensiones social, ambiental y económica, debe ser la misión de las universidades para encaminar a las sociedades hacia la sostenibilidad. La principal estrategia a destacar en el marco de la responsabilidad social y ambiental, radica en aportar al alcance de las metas de los Objetivos del Desarrollo Sostenible (Sureda et al., 2017). La transformación, adaptación y desempeño de nuevos roles en las recientes actividades de índole social, cultural y ambiental (Chumaceiro Hernández et al., 2018); requieren que la educación superior demuestre ser la herramienta clave en la formación de profesionales que respondan a los problemas ambientales y necesidades sociales con acciones coherentes a la sostenibilidad (Aznar Minguet et al., 2014).

Determinar el nivel de cultura ambiental en los estudiantes universitarios, implica conocer el grado de conocimientos sobre temas ambientales; el tipo de decisiones a aplicar cuando de ellas dependa la conservación ambiental. Finalmente considera; el nivel de inclusión de los conocimientos que tienen sobre el área ambiental y comportamiento positivo hacia la protección de los recursos, en el sistema de valores y creencias que poseen. Según Vacío (2017), el Índice de cultura Ambiental (ICA) se lo obtiene al promediar las ponderaciones de cada sección del instrumento en Wisconsin Environmental Literacy Survey; actitud, comportamiento y conocimiento. Estas mostrarán diferentes valoraciones que se calificarán acorde a la respuesta seleccionada por el encuestado, el ICA se obtiene de la suma de los tres subíndices, se promedian y transforman a la escala de $1-10$.

\section{METODOLOGÍA}

El diagnóstico del nivel de cultura ambiental se basó en evaluar los indicadores de actitud, comportamiento y conocimiento ambiental adquiridos en clases y en la vida cotidiana, a un total de 2366 encuestas dirigidas a los estudiantes matriculados en el 2020 de una institución de educación superior. Se aplicó el cuestionario sobre cultura ambiental mediante la adaptación del instrumento Wisconsin Environmental Literacy Survey modificado (Kibert, 2000), esta herramienta contó con cuatro secciones donde la persona encuestada reflejará su nivel de aceptación o rechazo acorde a las siguientes aspectos (IsaacMárquez, 2011): Datos generales (edad, género, facultad); Actitudes (predisposición para responder ante los problemas ambientales) con una escala numérica de totalmente de acuerdo, de acuerdo, sin opinión, desacuerdo, muy en desacuerdo. Intenciones de comportamiento (frecuencia con las que realizan acciones ambientales); con la escala numérica de casi siempre, a menudo, a veces, casi nunca, nunca. Y 
conocimientos ambientales (conceptos ecológicos básicos y conocimiento de la problemática ambiental).

\subsection{Evaluación de la cultura ambiental}

El cuestionario de cultura ambiental constó de 45 preguntas, actitudes (15 preguntas), comportamientos (15 preguntas) y conocimiento (15 preguntas); a cada respuesta se le asignará un valor de 4 a la respuesta más favorable y de 0 a la menos favorable. Esto con respecto a las secciones de actitud y comportamiento, cabe recalcar que algunas de las preguntas de cultura ambiental estarán redactadas de manera tal que las respuestas correctas tendrán mayor valor en un extremo de la escala y en otros casos en el otro extremo. En la sección de conocimientos se otorgará un valor de 4 a la respuesta correcta y 0 a las incorrectas (Isaac-Márquez, 2011).

La base de datos de las variables de estudio se la manejó en Microsoft Excel 2013 y mediante el programa estadístico SPSS V22. Se aplicó estadísticas descriptivas (barras, diagramas de sectores, resúmenes estadísticos), estadística inferencial no paramétrica ( $U$ - Mann Withman y Kusskal Wallis) y estadística multivariada (Análisis de Clúster jerárquico con el intervalo de correlación de Pearson). El cálculo del índice de cultura ambiental (ICA) se realizará acorde a la metodología propuesta por Vacío (2017), donde indica la suma de las ponderaciones de las tres secciones (actitud, comportamiento y conocimiento), por lo que el valor más alto será 180 de la suma final de las tres secciones. Se calculará la media total de los tres componentes y se transformará a escala 1-10, lo cual reflejará la siguiente escala de puntuaciones (ver Tabla 1).

Tabla 1. Interpretación de la escala de puntuaciones de cultura ambiental

\begin{tabular}{lll}
\hline Puntuación & Interpretación & \\
\hline $10,0-9,0$ & Excelente & \\
$8,9-8,0$ & Muy aceptable & \\
$7,9-7,0$ & Aceptable & \\
$6,9-6,0$ & Inaceptable & \\
$5,9-1,0$ & Nivel bajo & \\
\hline & Fuente: & (Vacio \\
& Fraga, 2017) &
\end{tabular}

2. RESULTADOS $\mathrm{Y}$

\section{DISCUSIÓN}

La muestra de estudiantes universitarios que se evaluó en Cultura Ambiental, consta un rango mínimo 17 años $\mathrm{y}$ máximo 43 años. El análisis de las edades de los estudiantes, indicó que los alumnos de 17, 18, 19, 20, 21, 22, 23, 24 años, son los de mayor incidencia en esta evaluación. Se observó que el $20 \%$ de estudiantes eran de 19 años, seguido por las edades de 18 y 20. El análisis de la frecuencia permitió la definición de la desviación estándar de 3,813, la media 20,94 , la mediana de 20 , percentil al $25 \%$ (19 años), percentil al 75\% (22 años) y moda de 19 (ver Figura 1). 


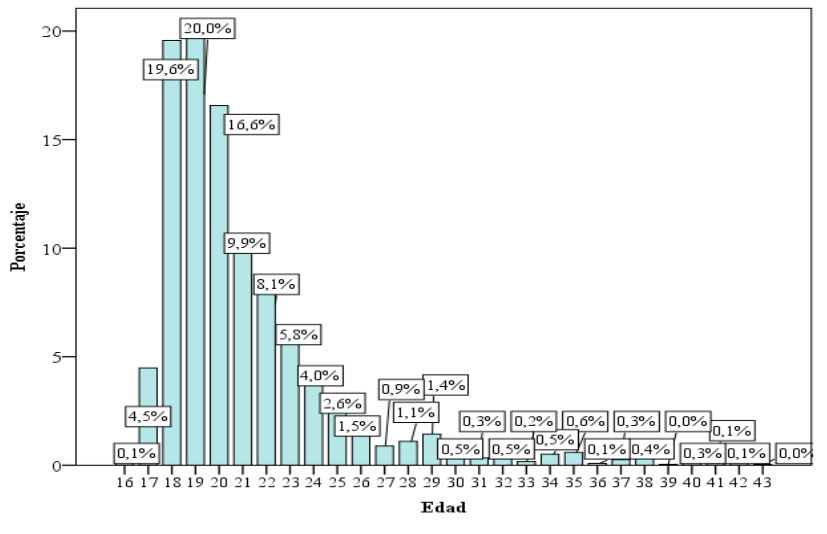

Figura 1. Edades de estudiantes

El análisis de la frecuencia de género, evidenció que la mayoría de los estudiantes encuestados de las diferentes facultades fueron mujeres $57,2 \%$ (1354) y el 42,8\% (1012) correspondió al género masculino. La definición de la desviación estándar 0,495, la media 1,43, la mediana (Femenino), moda (Femenino), percentil al 25\% (Femenino) y percentil al 75\% (Masculino) (ver Figura 2).

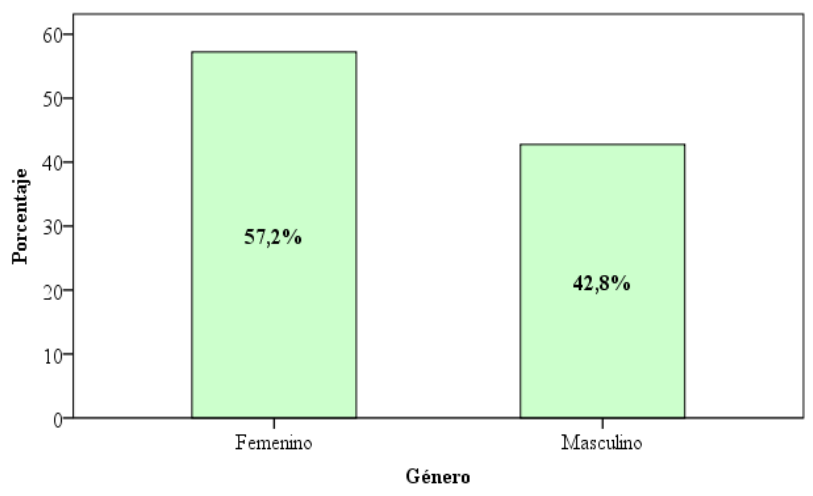

Figura 2. Frecuencia de Género en estudiantes

2.1. Análisis de frecuencia en la cultura ambiental

Este apartado presenta por cada aspecto de la cultura ambiental, actitud, comportamiento y conocimiento; los rangos obtenidos por cada pregunta, que demuestran mayor o menor incidencia.

\subsubsection{Actitud}

Los resultados de las actitudes se encontraron en un rango mínimo (0) y máximo (4), con una moda de totalmente de acuerdo en siete preguntas y de acuerdo en siete preguntas, en la pregunta sobre PA4 "Las regulaciones de contaminación en una comunidad no deberían interferir con el crecimiento industrial y desarrollo"; se encontró mayor incidencia, en indicar desacuerdo $(1,82)$. Con respecto a la media, se obtuvo la puntuación más alta $(2,77)$ en la pregunta PA13 "Me preocupan los riesgos para la salud ambiental, como los causados por la contaminación del aire o del agua"; como se evidencia en la Tabla 2 .

Tabla 2. Resultados de los estudiantes con respecto a las Actitudes

\begin{tabular}{|c|c|c|c|c|c|c|c|c|c|c|c|c|c|c|c|c|}
\hline \multicolumn{2}{|c|}{ Preguntas } & $\begin{array}{c}\text { PA } \\
1\end{array}$ & $\begin{array}{c}\text { PA } \\
2\end{array}$ & $\begin{array}{c}\text { PA } \\
3\end{array}$ & $\begin{array}{c}\text { PA } \\
4\end{array}$ & $\begin{array}{c}\mathrm{PA} \\
5\end{array}$ & $\begin{array}{c}\mathrm{PA} \\
6\end{array}$ & $\begin{array}{c}\text { PA } \\
7\end{array}$ & $\begin{array}{c}\text { PA } \\
8\end{array}$ & $\begin{array}{c}\text { PA } \\
9\end{array}$ & $\begin{array}{c}\text { PA } \\
10 \\
\end{array}$ & $\begin{array}{l}\text { PA } \\
11\end{array}$ & $\begin{array}{l}\text { PA } \\
12 \\
\end{array}$ & \begin{tabular}{|l} 
PA \\
13 \\
\end{tabular} & $\begin{array}{c}\text { PA } \\
14 \\
\end{array}$ & $\begin{array}{r}\text { PA } \\
15 \\
\end{array}$ \\
\hline \multicolumn{2}{|c|}{ Media } & $\begin{array}{c}2,1 \\
7\end{array}$ & $\begin{array}{c}2,4 \\
3\end{array}$ & $\begin{array}{c}2,3 \\
6\end{array}$ & $\begin{array}{c}1,8 \\
2\end{array}$ & $\begin{array}{c}2,6 \\
6\end{array}$ & $\begin{array}{c}2,4 \\
3\end{array}$ & $\begin{array}{c}2,2 \\
4\end{array}$ & $\begin{array}{c}2,5 \\
3\end{array}$ & $\begin{array}{c}2,6 \\
8\end{array}$ & 2,69 & 2,57 & 2,52 & 2,77 & 2,66 & 2,40 \\
\hline \multicolumn{2}{|c|}{ Moda } & 3 & 3 & 3 & 1 & 3 & 3 & 3 & 4 & 4 & 4 & 4 & 4 & 4 & 4 & 3 \\
\hline \multicolumn{2}{|c|}{$\begin{array}{c}\text { Desviación } \\
\text { estándar }\end{array}$} & $\begin{array}{l}1,4 \\
29\end{array}$ & $\begin{array}{l}1,2 \\
62\end{array}$ & $\begin{array}{l}1,3 \\
69\end{array}$ & $\begin{array}{l}1,2 \\
82 \\
\end{array}$ & $\begin{array}{l}1,2 \\
91\end{array}$ & $\begin{array}{l}1,3 \\
75\end{array}$ & $\begin{array}{l}1,3 \\
41 \\
\end{array}$ & $\begin{array}{l}1,4 \\
78\end{array}$ & $\begin{array}{l}1,4 \\
56\end{array}$ & $\begin{array}{c}1,41 \\
9\end{array}$ & $\begin{array}{c}1,37 \\
0\end{array}$ & $\begin{array}{c}1,46 \\
9\end{array}$ & $\begin{array}{c}1,38 \\
0 \\
\end{array}$ & $\begin{array}{c}1,32 \\
8\end{array}$ & $\begin{array}{c}1,23 \\
8\end{array}$ \\
\hline \multirow{2}{*}{$\begin{array}{c}\text { Percenti } \\
\text { les }\end{array}$} & $25 \%$ & 1 & 2 & 1 & 1 & 2 & 1 & 1 & 1 & 2 & 2 & 2 & 1 & 2 & 2 & 1 \\
\hline & $75 \%$ & 3 & 3 & 4 & 3 & 4 & 4 & 3 & 4 & 4 & 4 & 4 & 4 & 4 & 4 & 3 \\
\hline
\end{tabular}




\subsubsection{Conocimiento}

Con respecto al conocimiento de los estudiantes, se encontraron en un rango de respuestas mínimo (0) y máximo (4), con una media más alta 2,65 en la pregunta PCONO2 “Todos los mismos organismos individuales que viven en el suelo en un bosque, en particular comparten las características totales" indicaron conocer correctamente la definición de hábitat. La PCONO8 "La tasa de extinción de especies, es más alta ahora que en cualquier otro momento desde el periodo de extinción de los dinosaurios. La causa principal de esta rápida disminución de la biodiversidad es la alteración de los hábitats por las actividades del humano $(0,91)$; como se observa en la Tabla 3.

Tabla 3. Respuestas de los estudiantes con respecto al conocimiento

\begin{tabular}{|c|c|c|c|c|c|c|c|c|c|c|c|c|c|c|c|c|}
\hline \multicolumn{2}{|c|}{ Preguntas } & $\begin{array}{l}\mathrm{PCO} \\
\mathrm{NO} 1\end{array}$ & $\begin{array}{l}\mathrm{PCO} \\
\mathrm{NO} 2\end{array}$ & $\begin{array}{l}\mathrm{PCO} \\
\text { NO3 }\end{array}$ & $\begin{array}{l}\mathrm{PCO} \\
\mathrm{NO} 4\end{array}$ & $\begin{array}{l}\text { PCO } \\
\text { NO5 }\end{array}$ & $\begin{array}{l}\text { PCO } \\
\text { NO6 }\end{array}$ & $\begin{array}{l}\text { PCO } \\
\text { NO7 }\end{array}$ & $\begin{array}{l}\mathrm{PCO} \\
\text { NO8 }\end{array}$ & $\begin{array}{l}\text { PCO } \\
\text { NO9 }\end{array}$ & $\begin{array}{c}\text { PCON } \\
\text { O10 }\end{array}$ & $\begin{array}{c}\text { PCON } \\
\text { O11 }\end{array}$ & $\begin{array}{c}\text { PCON } \\
\text { O12 }\end{array}$ & $\begin{array}{c}\text { PCON } \\
\text { O13 }\end{array}$ & $\begin{array}{c}\text { PCON } \\
\text { O14 }\end{array}$ & $\begin{array}{c}\text { PCON } \\
\text { O15 }\end{array}$ \\
\hline \multicolumn{2}{|c|}{ Media } & 1,02 & 2,65 & 1,67 & 1,49 & 2,09 & 1,83 & 1,16 & 0,91 & 2,23 & 1,44 & 1,03 & 1,27 & 1,22 & 0,98 & 1,91 \\
\hline \multicolumn{2}{|c|}{ Moda } & 0 & 4 & 0 & 0 & 4 & 0 & 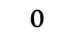 & 0 & 4 & 0 & 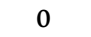 & 0 & 0 & 0 & 0 \\
\hline \multicolumn{2}{|c|}{$\begin{array}{c}\text { Desviación } \\
\text { estándar }\end{array}$} & 1,745 & 1,892 & 1,974 & 1,935 & 1,999 & 1,993 & 1,816 & 1,680 & 1,987 & 1,920 & 1,752 & 1,862 & 1,843 & 1,721 & 1,998 \\
\hline \multirow{2}{*}{$\begin{array}{l}\text { Perce } \\
\text { ntiles }\end{array}$} & $25 \%$ & 0 & 0 & 0 & 0 & 0 & 0 & 0 & 0 & 0 & 0 & 0 & 0 & 0 & 0 & 0 \\
\hline & $75 \%$ & 4 & 4 & 4 & 4 & 4 & 4 & 4 & 0 & 4 & 4 & 4 & 4 & 4 & 0 & 4 \\
\hline
\end{tabular}

Fuente: Elaboración propia (2020)

\subsubsection{Comportamiento}

El comportamiento en los universitarios, según los resultados indicaron un rango de respuesta mínimo (0) y máximo (4), la media con puntuación más alta $(2,96)$ en la pregunta PCOMP1 "Apago las luces y los electrodomésticos cuando no se utilizan para ahorrar electricidad"; en la cual los estudiantes eligieron casi siempre tomar acciones por reducir el consumo energético. La media más baja se presentó en la PCOMP14 "Envío cartas al periódico sobre problemas $\mathrm{O}$ problemas ambientales" indicaron no enviar o denunciar algún caso de contaminación ambiental a los medios de comunicación, ver Tabla 4.

Tabla 1. Resultados de los estudiantes con respecto al comportamiento

\begin{tabular}{|c|c|c|c|c|c|c|c|c|c|c|c|c|c|c|c|c|}
\hline \multicolumn{2}{|c|}{ Preguntas } & $\begin{array}{l}\text { PCO } \\
\text { MP1 }\end{array}$ & $\begin{array}{l}\text { PCO } \\
\text { MP2 }\end{array}$ & $\begin{array}{l}\text { PCO } \\
\text { MP3 }\end{array}$ & $\begin{array}{l}\text { PCO } \\
\text { MP4 }\end{array}$ & $\begin{array}{l}\text { PCO } \\
\text { MP5 }\end{array}$ & $\begin{array}{l}\text { PCO } \\
\text { MP6 }\end{array}$ & $\begin{array}{l}\text { PCO } \\
\text { MP7 }\end{array}$ & $\begin{array}{l}\text { PCO } \\
\text { MP8 }\end{array}$ & $\begin{array}{l}\text { PCO } \\
\text { MP9 }\end{array}$ & $\begin{array}{c}\text { PCO } \\
\text { MP1 } \\
0 \\
\end{array}$ & $\begin{array}{c}\text { PCO } \\
\text { MP1 } \\
1 \\
\end{array}$ & $\begin{array}{c}\text { PCO } \\
\text { MP1 } \\
2 \\
\end{array}$ & $\begin{array}{c}\text { PCO } \\
\text { MP1 } \\
3 \\
\end{array}$ & $\begin{array}{c}\text { PCO } \\
\text { MP1 } \\
4 \\
\end{array}$ & $\begin{array}{c}\text { PCO } \\
\text { MP1 } \\
5 \\
\end{array}$ \\
\hline \multicolumn{2}{|c|}{ Media } & 2,96 & 2,55 & 2,42 & 2,52 & 2,35 & 2,40 & 2,25 & 2,37 & 2,26 & 2,21 & 1,44 & 2,03 & 2,16 & 1,14 & 1,18 \\
\hline \multicolumn{2}{|c|}{ Moda } & 4 & 2 & 2 & 2 & 2 & 4 & 2 & 2 & 2 & 2 & 0 & 2 & 2 & 0 & 0 \\
\hline \multicolumn{2}{|c|}{$\begin{array}{c}\text { Desviación } \\
\text { estándar }\end{array}$} & $\begin{array}{c}1,20 \\
8 \\
\end{array}$ & $\begin{array}{c}1,10 \\
5\end{array}$ & $\begin{array}{c}1,25 \\
5\end{array}$ & $\begin{array}{c}1,07 \\
7 \\
\end{array}$ & $\begin{array}{c}1,18 \\
5\end{array}$ & $\begin{array}{c}1,33 \\
0 \\
\end{array}$ & $\begin{array}{c}1,24 \\
5\end{array}$ & $\begin{array}{c}1,21 \\
4 \\
\end{array}$ & $\begin{array}{c}1,18 \\
1\end{array}$ & 1,228 & 1,361 & 1,211 & 1,194 & 1,325 & 1,355 \\
\hline \multirow{2}{*}{$\begin{array}{l}\text { Percen } \\
\text { tiles }\end{array}$} & $\begin{array}{r}25 \\
\% \\
\end{array}$ & 2 & 2 & 2 & 2 & 2 & 1 & 1 & 2 & 1 & 1 & 0 & 1 & 1 & 0 & 0 \\
\hline & $\begin{array}{r}75 \\
\%\end{array}$ & 4 & 3 & 4 & 3 & 3 & 4 & 3 & 3 & 3 & 3 & 2 & 3 & 3 & 2 & 2 \\
\hline
\end{tabular}




\subsection{Análisis de Clúster de Cultura Ambiental en estudiantes universitarios}

En el análisis de Clúster, se observó las correlaciones más fuertes en la sección de Actitud, con las preguntas PA9 "Se debe reservar más tierra para los hábitats de vida silvestre" y PA10 "Me preocupa la cantidad de desechos que se producen en este país", se encuentran correlacionadas debido a la valoración alta obtenida en la media (6,68 y 6,69 respectivamente); al igual que Igualmente se visualizó una relación entre las preguntas PA13 "Me preocupan los riesgos para la salud ambiental, como los causados por la contaminación del aire o del agua" y PA14 "Creo que puedo contribuir a la solución de problemas ambientales con mis acciones" que obtuvieron las valoraciones altas en los promedios totales ( 2,77 y 2,66 respectivamente). Observar figura 3.

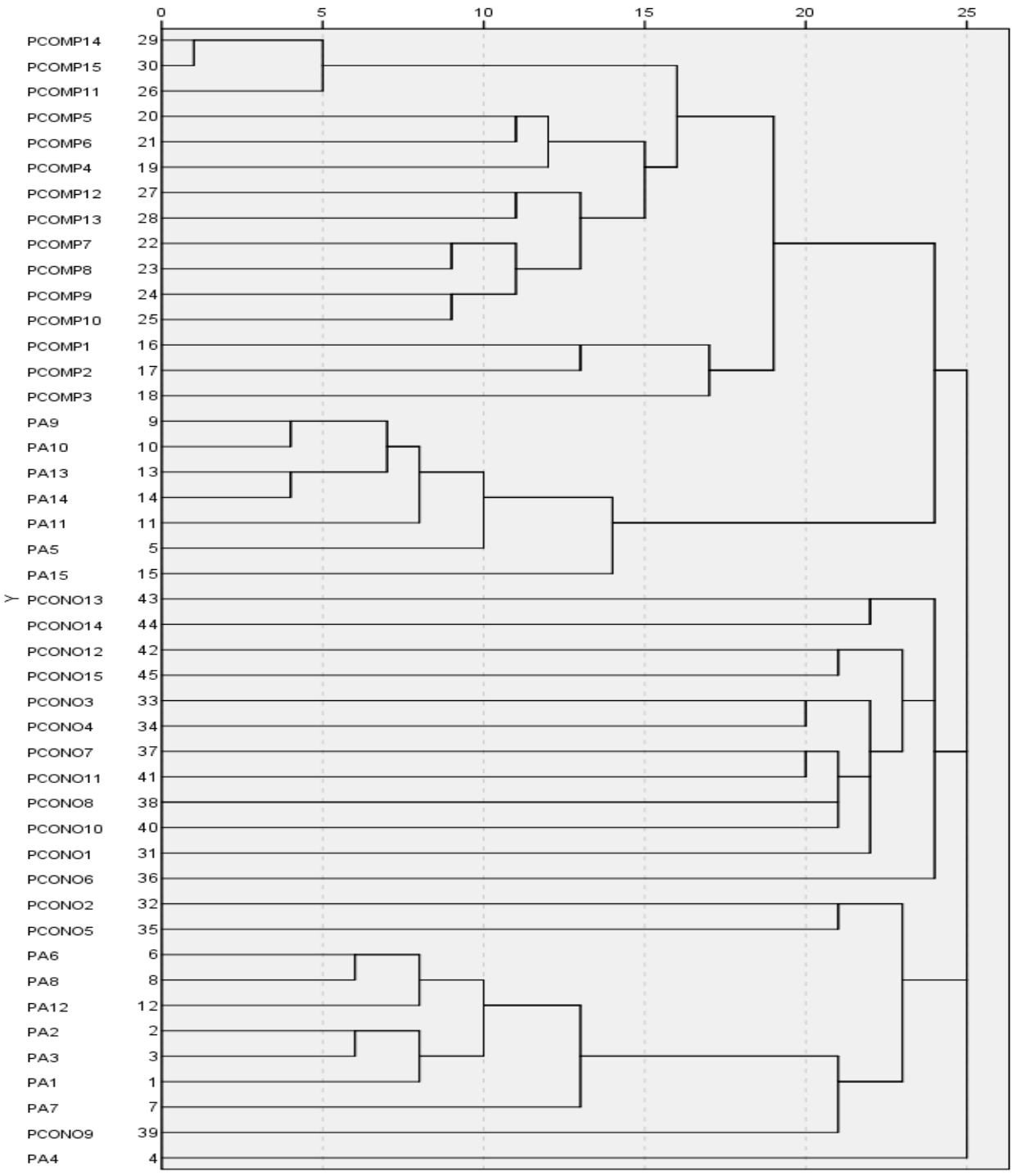

Figura 3. Dendograma del cuestionario de cultura ambiental en estudiantes universitarios Fuente: Elaboración propia, (2020) 
Y finalmente se presentó una correlación de las preguntas de comportamiento entre PCOMP14 "Envío cartas al periódico sobre problemas o problemas ambientales", PCOMP15 "He denunciado problemas ambientales o violaciones que he notado a las autoridades correspondientes" y PCOMP11 "Escribo o llamo a los políticos para expresar mis puntos de vista sobre cuestiones ambientales". Por el puntaje bajo que obtuvieron en la media total; 1,$14 ; 1,18$ y 1,44 respectivamente. Con lo que se puede concluir que los estudiantes encuestados, no realizan acción alguna para denunciar las negligencias ambientales por parte de políticos, ciudadanos e industrias, tampoco las hacen conocer a los medios de comunicación ya que muestran poco interés por remediar, mitigar los impactos ambientales por medio de las demandas y son muy pocos los que presentan medidas de soluciones con temáticas ambientales a las autoridades competentes. No obstante, no se evidenció correlación alguna en los resultados obtenidos de las preguntas de conocimiento.

\subsection{Análisis de relación entre variables de estudio}

La determinación de las relaciones significativas entre las variables consideradas en el estudio, permiten especificar las incidencias existentes con las variables del estudio (Género, edad y facultad) con las variables que incluye la cultura ambiental (Actitud, Conocimiento y Comportamiento).

\subsubsection{Género y Actitud}

El factor género se encontró influenciada en las actitudes de los estudiantes, ya que se determinó una diferencia estadística en los resultados (UMW $=626490,50 ; \mathrm{p}$ valor $=0,00)$. Del total de estudiantes encuestados con respecto a las actitudes que tienen las personas para reflejar sus sentimientos hacia la naturaleza, se puede apreciar que las mujeres obtuvieron una media de 6,25 superior a los hombres 6,02 . Lo que evidencia que las mujeres optan por expresar más aprecio o aceptabilidad hacia el medio ambiente que los hombres, pero ambos reflejan actitudes inaceptables (ver Figura 4). La selección exclusiva de estudiantes con puntuación $(7,0$ - 10), indicó que un total de $544(23 \%)$ mujeres y $339(14,33 \%)$ hombres con actitudes aceptables.

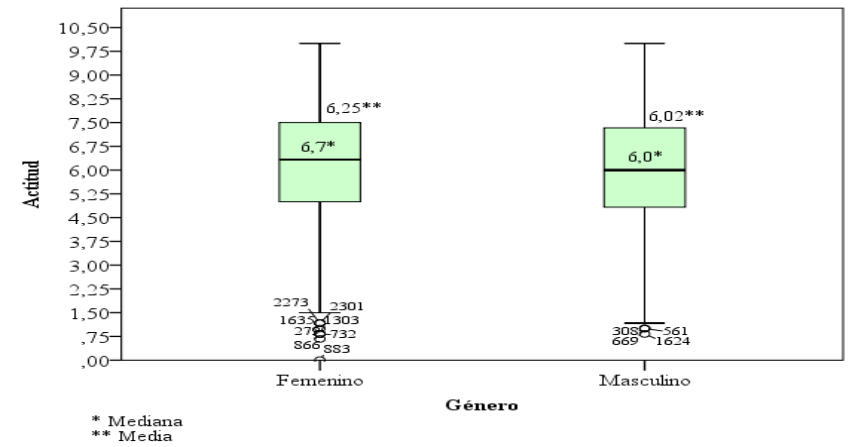

Figura 4. Diagrama de cajas entre el género y la actitud de estudiantes

\subsubsection{Género y Comportamiento}

El comportamiento de los estudiantes se encontró influenciada por el género, debido que existió diferencia estadística en los resultados (UMW $=634661,500$; p-valor $=0,002)$. El comportamiento de los hombres fue mayor con 5,5 de promedio que el de las mujeres que fue 5,3 . Se seleccionó únicamente a estudiantes que obtuvieron un promedio $(7,0$ - 10) que es de categoría aceptable. 
Con un total de $224(9,47 \%)$ del sexo femenino y $234 \quad(9,89 \%)$ del sexo masculino, indica que los estudiantes tienen acciones más positivas hacia la naturaleza (ver Figura 5).

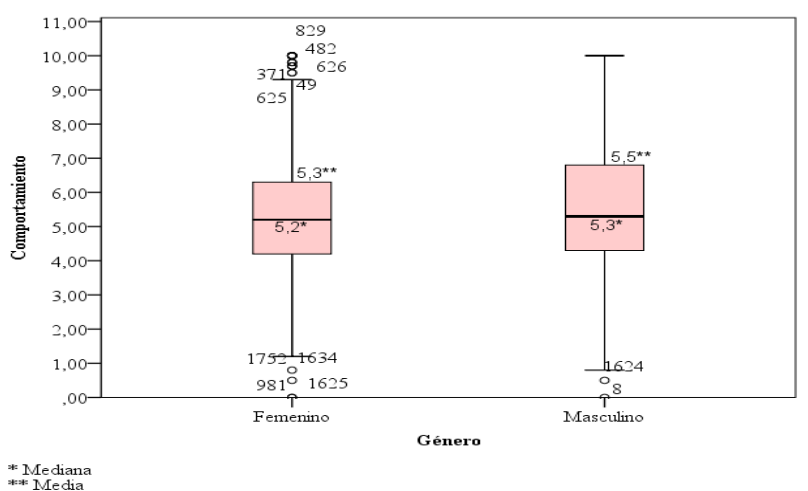

Figura 5. Diagrama de cajas entre el género y el comportamiento de los estudiantes

\subsubsection{Género y Conocimientos}

El factor género no determinó variación en los conocimientos de los estudiantes, debido a la similitud estadística en los resultados (UMW = 676957,000; p-valor $=0,617)$. El conocimiento varones $\mathrm{y}$ mujeres tienen la misma media $(3,8)$; por ende, a pesar de ser estudiantes del tercer nivel ambos presentan un nivel muy bajo en conocimientos de temática ambiental (ver Figura 6).

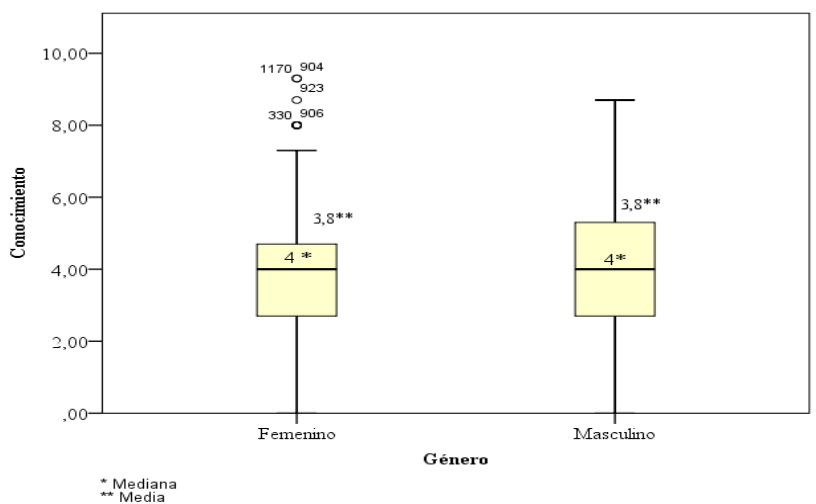

Figura 6. Diagrama de cajas entre el género y el conocimiento de los estudiantes

\subsubsection{Edad y Actitud}

Los estudiantes de 17, 18, 19, 20, 21, 22, 23, 24 años tienen mayor incidencia que las demás edades (figura 6). Se consideró sólo estas edades para el análisis de estadística no paramétrica. Del total de estudiantes se seleccionó a 2092, se seleccionó a los estudiantes que obtuvieron un promedio $(10-7,0)$ de categoría aceptable. Se identificó un total 35 estudiantes de 17 años (1,48\%), 160 alumnos de 18 años (6,76\%), 166 (7,02\%) en estudiantes de 19 años, $130(5,49 \%)$ en alumnos de 20 años, 94 (3,97\%) en estudiantes de 21 años, $74(3,13 \%)$ en alumnos de 22 años, 58 (2,45\%) en estudiantes de 22 años y $43(1,82 \%)$ en alumnos de 24 años de edad.

La actitud de estudiantes no se encontró influenciada por el factor edad, debido a diferencia estadística en los resultados $(\mathrm{KW}=13,497 ; \mathrm{p}$-valor $=0,061) . \quad$ Los estudiantes de mayor edad 23 y 24 años obtuvieron una media más alta de 6,4 indicando que estudiantes a esa edad se encuentran cursando los últimos semestres y por ende su sensibilización hacia el medio ambiente es un poco positiva y se apreció a la edad de 20 años el promedio más bajo de actitud hacia el ambiente (ver Figura 14). 


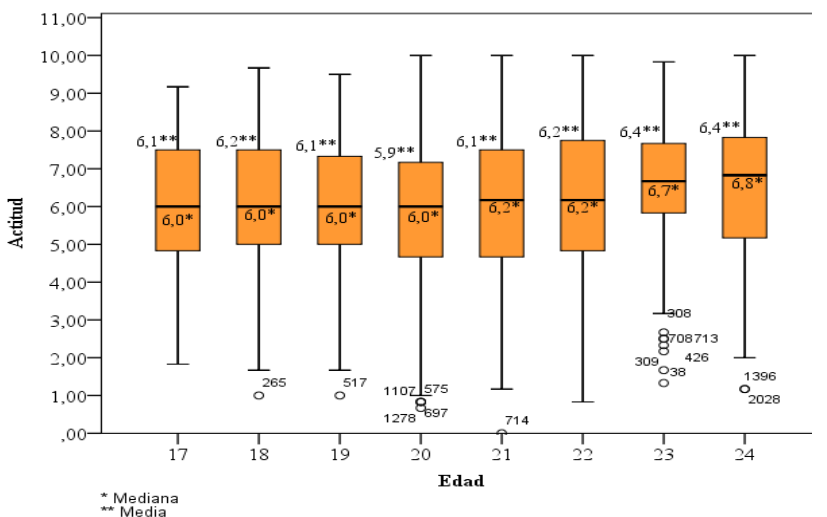

Figura 6. Diagrama de caja entre la edad y la actitud de los estudiantes

\subsubsection{Edad y Comportamiento}

Los rangos entre edades fueron mínimos de 17 años y máximo de 24 años, a partir de la muestra de estudiantes (2092) seleccionada, se seleccionó a los estudiantes que obtuvieron un promedio $(10-7,0)$ de categoría aceptable. Dando como resultado, un total $22(0,93 \%)$ en estudiantes de 17 años, $68(2,87 \%)$ en alumnos de 18 años, $98(4,14 \%)$ en estudiantes de 19 años, $72(3,04 \%)$ en alumnos de 20 años, 54 (2,28\%) en estudiantes de 21 años, $46(1,94 \%)$ en alumnos de 22 años, 30 (1,27\%) en estudiantes de 23 años y $21(0,89 \%)$ en alumnos de 24 años de edad.

La edad es un factor que determinó cambios en los comportamientos de los estudiantes, debido a que no existió significancia estadística en los resultados $(\mathrm{KW}=14,680 ; \mathrm{p}$-valor $=0,040)$. Se pudo observar que los estudiantes de 22 años obtuvieron una media más alta de 5,7, seguido de 24 y 19 años con promedio de 5,5 que a pesar de tener la media más alta entre las edades seleccionadas su puntuación indica que sus comportamientos tienen un nivel bajo (ver Figura 7).

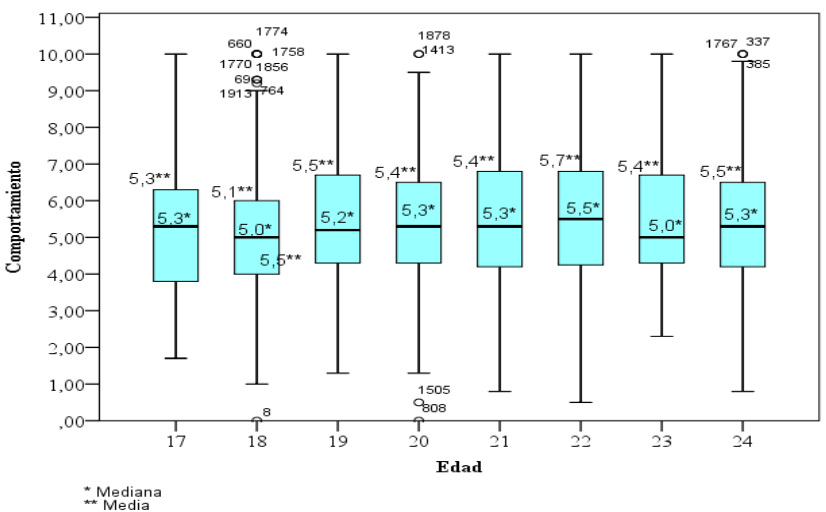

Figura 7. Diagrama de caja entre Edad y el comportamiento de los estudiantes

\subsubsection{Edad y Conocimiento}

Los estudiantes seleccionados (2092) para este análisis estuvieron entre los rangos mínimo de 17 años y máximo de 24 años, acorde a sus respuestas se procedió a clasificar solo a los estudiantes que obtuvieron un promedio $(10-7,0)$ que es hasta la categoría aceptable. Se identificó un total $2(0,08 \%)$ en estudiantes de 17 años, $13(0,55 \%)$ en alumnos de 18 años, $10(0,42 \%)$ en estudiantes de 19 años, 13 $(0,55 \%)$ en alumnos de 20 años, 8 $(0,34 \%)$ en estudiantes de 21 años, 2 $(0,08 \%)$ en alumnos de 22 años, 6 $(0,25 \%)$ en estudiantes de 23 años y 3 $(0,13 \%)$ en alumnos de 24 años de edad.

El conocimiento de los estudiantes no es influenciado por la edad, a causa de que la existió significancia estadística (KW= 0,675; p-valor $=0,999)$. Se determinó que una media de 3,9 los estudiantes de 20 y 24 años obtuvieron el puntaje más alto entre las edades analizadas; es decir, que se encuentra una mayor recepción de conocimientos ambientales que el resto de edades. Adicionalmente, se visualizó que los estudiantes de 17 años que están cursando alrededor de nivelación y primeros semestres tienen más bajo su nivel de conocimiento; por tal es 
justificable debido que sus conceptualizaciones ambientales son muy limitadas influenciadas por la educación secundaria recibida (ver Figura 8).

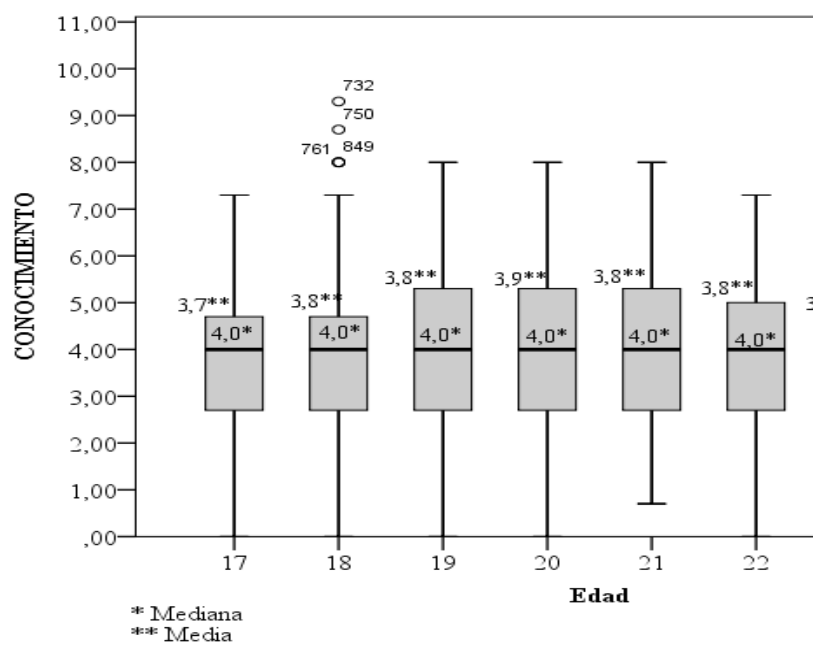

Figura 8. Diagrama de cajas entre la edad y el conocimiento de los estudiantes

\subsubsection{Facultad y Actitud}

Las actitudes de los estudiantes se vieron influenciadas por las facultades (campos del conocimiento), debido que en los resultados existió diferencias estadísticas $(\mathrm{KW}=151,432 ; \mathrm{p}$-valor $=0,000)$. Con respecto a las actitudes que poseen los estudiantes por facultad, se reflejó que estudiantes de la Unidad de Estudios a Distancia, Enfermería y la Facultad de Ciencias Ambientales; poseen los promedios más altos $(6,8 ; 6,8 ; 6,3$ respectivamente) con respecto a la comparación entre las ocho facultades. Sin embargo, estas medias las categorizan en un nivel inaceptable. La Facultad de Ciencias Pecuarias $(5,5)$ y FCI $(5,7)$ tienen las medias más bajas al no brindar la importancia, el respeto y amor por la naturaleza, evidenciando un nivel bajo en actitudes (ver Figura 9).
De los 2366 estudiantes, se consideró a los estudiantes que obtuvieron mayor puntaje $(10$ - 7,0), se observó de la Facultad de Ciencias Agracias 86 (3,63\%) alumnos, Facultad de Ciencias Ambientales 112 $(4,73 \%)$, Unidad de Estudios a Distancia 133 (5,62\%), Facultad de Ciencias Empresariales 120 (5,07\%), carrera de Enfermería 162 (6,85\%), Facultad de Ciencias de la Ingeniería 93 (3,93\%), Facultad de Ciencias Pecuarias 66 (2,79\%) y Nivelación 112 (4,73\%). Se reflejó igual un índice muy bajo de estudiantes con actitud aceptable.

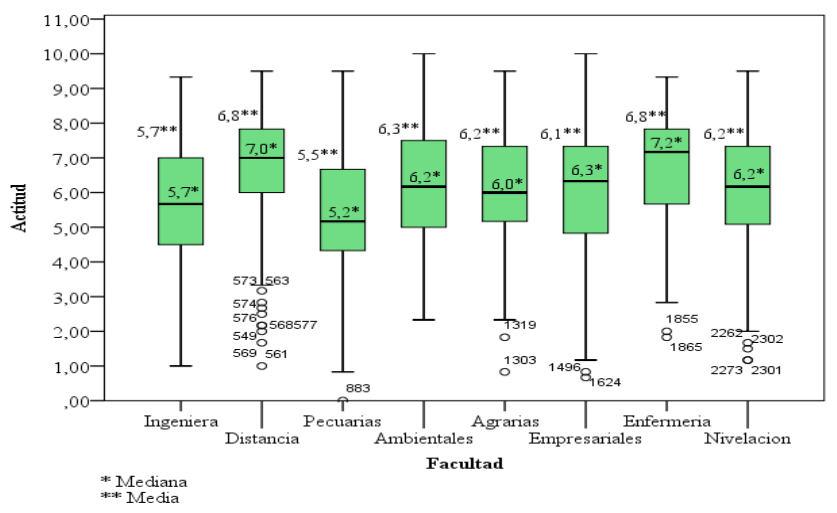

Figura 9. Diagrama de cajas entre las facultades y la actitud de los estudiantes

\subsubsection{Facultad y Comportamiento}

Las facultades influyen en los comportamientos de los estudiantes, debido que existió diferencias estadísticas $(\mathrm{KW}=21,854 ; \mathrm{p}$-valor $=0,003)$. Las Facultad de Ciencias Ambientales $(5,6)$, Facultad de Ciencias Pecuarias $(5,5)$, Facultad de Ciencias Agrarias (5,5); al ser carreras que tienen más contacto directo con la naturaleza, las acciones hacia el cuidado del medio ambiente son mayores al resto de facultades. Sin embargo, sus promedios indican que sus 
comportamientos son nivel bajo. Además, se observó que la Facultad de Ciencias Empresariales $(5,0)$ obtuvo el promedio más bajo categorizado también con nivel bajo, debido que el enfoque en los resultados de aprendizajes de las carreras de esta facultad, se direccionan al crecimiento y control económico más no enfocados al desarrollo sostenible (ver Figura 10). Del total de estudiantes encuestados, se proyectó el número de estudiantes que se encuentran en el rango de 10 a 7,0, en puntuación acorde a cada facultad. Se evidenció que la Facultad de Ciencias Agracias tiene 57 (2,41\%) alumnos, Facultad de Ciencias Ambientales 74 (3,13\%), Unidad de Estudios a Distancia 43 (1,82\%), Facultad de Ciencias Empresariales 48 (2,03\%), carrera de Enfermería 39 (1,65\%), Facultad de Ciencias de la Ingeniería 76 $(3,21 \%)$, Facultad de Ciencias Pecuarias $61(2,58 \%)$ y Nivelación 60 (2,54\%), reflejando igual un índice muy bajo de estudiantes con comportamientos aceptable hacia el medio ambiente.

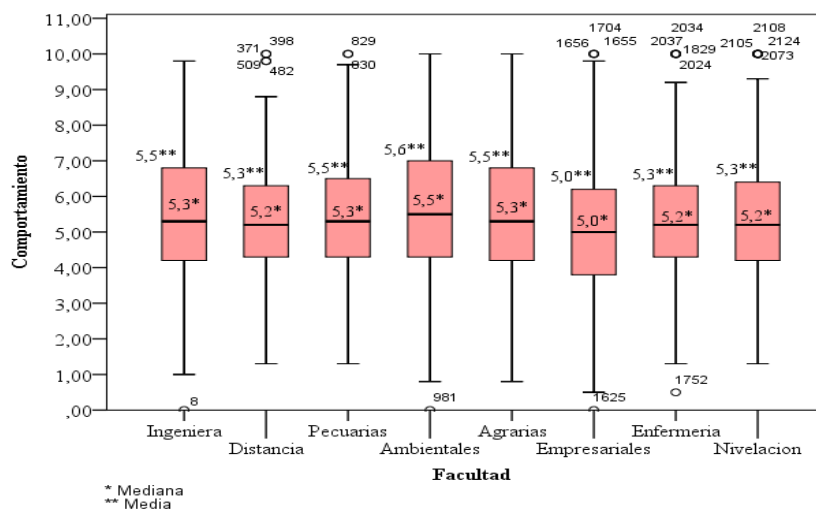

Figura 10. Diagrama de cajas entre las facultades y el comportamiento de los estudiantes

\subsubsection{Facultad y Conocimiento}

El conocimiento de los estudiantes se encontró influenciado por las facultades, debido que existió diferencias estadísticas en los resultados ( $\mathrm{KW}=210,362$; p-valor $=0,000)$. El conocimiento de los estudiantes influye mucho en la impartición de cada una de las materias establecidas dentro de las mallas académicas; por ende, se apreció que la Facultad de Ciencias Ambientales con 4,8 obtuvo entre facultades el promedio más alto. Sin embargo, esta puntuación la categoriza en nivel bajo. Con las medias más bajas están Nivelación $(3,1)$ y la Facultad de Ciencias Empresariales $(3,3)$ de igual manera con una categoría de nivel bajo (ver Figura 11). Se identificó a los alumnos que obtuvieron mayor puntuación $(10-7,0)$ en conocimientos de temática ambiental. En la Facultad de Ciencias Agracias se encontró 5 (0,21\%) alumnos, Facultad de Ciencias Ambientales 32 (1,35\%), Unidad de Estudios a Distancia $7(0,30 \%)$, Facultad de Ciencias Empresariales 2 (0,08\%), carrera de Enfermería 1 (0,04\%), Facultad de Ciencias de la Ingeniería 8 (0,34\%), Facultad de Ciencias Pecuarias 7 (0,30\%) y Nivelación $2(0,08 \%)$, reflejando igual un índice muy bajo de estudiantes con conocimientos aceptables hacia el medio ambiente.

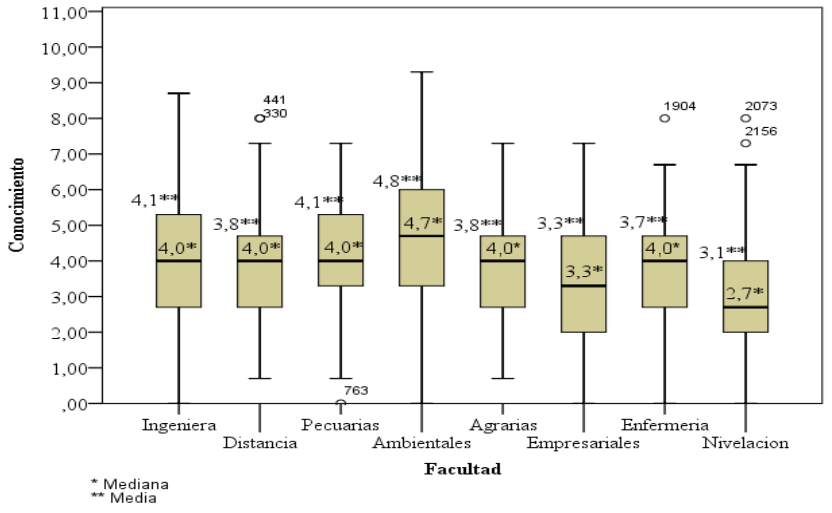

Figura 4. Diagramas de caja entre las facultades y el 
conocimiento de los estudiantes

La comparación entre facultades reflejó que la Facultad de Ciencias Ambientales obtuvo 5,6; seguido de la facultad de estudios a distancia 5,3 y la carrera de Enfermería 5,3. De acuerdo a la escala de puntuaciones se refleja un nivel bajo de cultura ambiental, a pesar de eso tienen las puntuaciones con mejor promedio debido a su malla curricular que están más relacionadas a la educación ambiental. Al igual que las puntuaciones más bajas recaen en la Facultad de Ciencias Empresariales obtuvo la calificación más baja 4,8; seguida de la Unidad de Admisión y Nivelación 4,9. El Índice de Cultura Ambiental (ICA) es de nivel bajo, siendo más drástico con puntuaciones inferiores en las facultades que menos imparten temas ambientales, corroborando que las clases influyen de manera directa en los estudiantes.

De los 2366 estudiantes encuestados sólo $10(0,42 \%)$ tienen una cultura ambiental muy aceptable, 86 (3,63\%) están en el rango de aceptables, 370 (15,64\%) alumnos se categorizaron de cultura inaceptable y el resto por obtener un rango inferior a 6 , que representa un nivel bajo. El $80,30 \%$ de estudiantes de la universidad tienen escasas actitudes, comportamientos y conocimientos ambientales; por lo que se requiere de medidas urgentes.

\section{4. Índice de cultura ambiental}

La valoración respectiva a cada indicador de actitud, comportamiento y conocimiento se reflejó en calificar de 4 a la respuesta más favorable y de 0 a la menos favorable al medio ambiente. Mediante la suma y promedio se determinó el ICA por facultad (ver Tabla 5). La evaluación fue realizada a estudiantes de primer semestre hasta décimo semestre respectivamente, de manera aleatoria en cada una de las facultades, en el caso de la carrera de enfermería se la consideró como facultad, debido que recién se está creando el ámbito de la medicina en la universidad por tal motivo aún no definen la facultad.

Tabla 5. ICA por facultad

\begin{tabular}{lcc}
\hline Estamento & Facultad & ICA \\
\hline & Ingeniería & 5,1 \\
& A Distancia & 5,3 \\
& Pecuarias & 5,0 \\
Estudiantes & Ambientales & 5,6 \\
& Agrarias & 5,1 \\
& Empresariales & 4,8 \\
& Enfermería & 5,3 \\
& Nivelación & 4,9 \\
\hline \multicolumn{2}{c}{ Total promedio } & 5,1 \\
\hline
\end{tabular}

El análisis estadístico determinó que la cultura ambiental en estudiantes universitarios es de nivel bajo. El análisis del cuestionario reflejó que estudiantes incidieron en obtener la media más baja en la PA4 indicando estar desacuerdo lo que es desfavorable para el ambiente, ya que se debe interferir para el desarrollo de una comunidad primero debe estar territorialmente organizado. Las industrias deberán ubicarse lejos de la comunidad y que regulen los procesos industriales con el fin de evitar afectaciones ambientales y de salud, con respecto a los comportamientos coincidieron en la PCOMP14 donde al igual obtuvieron una calificación baja indicando lamentablemente nunca enviar algún tipo de carta o queja algún periódico sobre los problemas ambientales. Por el 
contrario, un aspecto positivo se vio reflejado en la PCOMP1 donde los estudiantes inciden casi siempre en ahorrar electricidad.

Tabla 6. Valoración de la cultura ambiental

\begin{tabular}{lc}
\hline & Estudiantes \\
\hline Actitudes & 6,20 \\
Comportamientos & 5,40 \\
Conocimientos & 3,80 \\
\hline
\end{tabular}

Los estudiantes presentaron mayor puntuación en actitudes ambientales aun así estas son inaceptables, el comportamiento y el conocimiento ambiental de los alumnos fue nivel bajo (ver tabla 6), por lo que se requiere de mayor capacitación, preparación e inclusión de educación ambiental en la comunidad universitaria.

La cultura ambiental según (Vacio Fraga, 2017), llevada a cabo en México indicó; que los alumnos obtuvieron una calificación alta en actitudes ambientales 7,8 , pero baja en los componentes de comportamientos 4,7 y conocimientos ambientales 4,3. El ICA evidenció un resultado de 5,6 de una cultura ambiental reprobatoria. Por la poca preparación de los docentes en el ámbito ambiental; los estudiantes no reciben la educación ambiental como prioridad, concordando con (Perez de Villa Amil et al., 2017), de la Universidad de Cienfuegos, que demostraron que los docentes universitarios aún no comprenden la responsabilidad social, ambiental que tiene la universidad cubana actual y los retos que debe asumir en la educación ambiental para transmitirlo a estudiantes, trabajadores y comunidad.
Ante aquello, la investigación reflejó que los resultados de cultura ambiental en la universidad obtuvieron cierta similitud, pues los estudiantes obtuvieron bajo puntuación en conocimientos 3,8 y comportamientos 5,4 y un valor más elevado en actitudes 6,2 permitiendo analizar que los docentes pueden influir de manera directa en el pensar y actuar de los estudiantes y debido a su poca responsabilidad social - ambiental y preparación en el ámbito ambiental impiden que impartan idóneamente materias relacionadas a la Educación Ambiental, por lo que se vio reflejada la carencia de cultura ambiental de los alumnos. Según el estudio de (Levine \& Strube, 2012), descubrieron que los hombres tenían más conocimientos que las mujeres sobre cuestiones ambientales; los estudiantes mayores tenían actitudes ambientales implícitas y explícitas más favorables. Resultados similares se obtuvieron en la investigación, donde se observó que la edad está influenciada en las actitudes y comportamientos de los estudiantes con mayor edad; con respeto al género no influye en el conocimiento ambiental, contradiciéndose al estudio de Levine y Strube (2012), debido que no siempre el tipo de género influenciará en el conocimiento adquirido en las aulas. 


\section{CONCLUSIONES}

El análisis de relación entre las variables, evidenció que las actitudes de los estudiantes se vieron influenciadas por el género y las facultades, mas no por la edad. El comportamiento de los estudiantes es influenciado tanto por el género, la edad y las facultad a la que pertenece. $\mathrm{Y}$ el nivel de conocimiento ambiental universitario; se influenció únicamente por la facultad. Las asignaturas impartidas en las aulas deben incluir resultados de aprendizajes vinculados con los Objetivos del Desarrollo Sostenible, para incrementar la cultura ambiental en los futuros profesionales.

Es urgente liderar la adaptación de los currículos con enfoque a la sostenibilidad, mediante la inclusión de competencias transversales que incluyan los Objetivos de Desarrollo Sostenible. Puesto que se evidenció que la escasa cultura ambiental influye negativamente en el incremento de la sostenibilidad universitaria, pues se evidenció que los estudiantes llegan con buena actitud ambiental, pero la pierden en el avance de los semestres. Esta realidad se refuerza al obtener resultados donde se destaca que en las Facultades de Ciencias Pecuarias e Ingenierías poseen menos actitud ambiental; en la Facultad de Ciencias Empresariales el comportamiento no es amigable con el ambiente; así como, Nivelación y la facultad de Ciencias Empresariales se encuentran muy escasos de conocimientos ambientales.

El cuestionario analizado permitió concluir como el profesorado influye en ciertas actitudes y comportamientos de los estudiantes, no es posible incrementar la cultura ambiental en las aulas si los docentes no se someten a constante preparación con respecto al área ambiental. Por lo cual, si se incrementa la cultura ambiental que está basada en los hábitos, costumbres, acciones, valores, creencias y conocimiento de la comunidad universitaria enfocados a la protección, conservación y cuidado al medio ambiente, la carencia de concientización, educación ambiental, poca participación y la falta de sensibilización son las que reducen las posibilidades de que se incremente la sostenibilidad universitaria. 


\section{REFERENCIAS}

[1]. Alba Hidalgo, D. (2017). Hacia una fundamentación de la sostenibilidad en la educación superior. Revista Iberoamericana de Educación, 73, 15-34.

[2]. Aznar Minguet, P., Ull, M. A., Piñero, A., \& Martínez-Agut, M. P. (2014). La sostenibilidad en la formación universitaria: Desafíos y oportunidades. Educacion XX1, $17(1)$ 133-158. https://doi.org/10.5944/educxx1.1 7.1.10708

[3]. Chumaceiro Hernández, A., Hernández G de Velazco, J. J., \& Chirinos Noroño, E. J.; (2018). Responsabilidad social Universitaria, desarrollo sostenible y ciudadanía ambiental. Researchgate, 4(June), 53-64.

[4]. Chwialkowska, A., Bhatti, W. A., \& Glowik, M. (2020). The influence of cultural values on proenvironmental behavior. Journal of Cleaner Production, 122305. https://doi.org/10.1016/j.jclepro.2 020.122305

[5]. Hidalgo D., A. (2017). Hacia una fundamentación de la sostenibilidad en la educación superior. Iberoam, 0, 15-34.

[6]. Isaac-Márquez, R. (2011). Cultura ambiental en estudiantes de bachillerato . Estudio de caso de la educación ambiental en el nivel medio superior de Campeche Environmental Culture in HighSchool Students . Case Study of Environmental Education at the High-School Level in Campeche.
Revista Electrónica de Investigación Educativa, 13(2).

[7]. Levine, D. S., \& Strube, M. J. (2012). Environmental attitudes, knowledge, intentions and behaviors among college students. Journal of Social Psychology, 152(3), 308-326. https://doi.org/10.1080/00224545. 2011.604363

[8]. Perez de Villa Amil, Y., Bravo Montano, N., \& Valdés Méndez, I. (2017). Cultura Ambiental en los profesores Universitarios. Universidad y Sociedad, 9(2), 313-318.

[9]. Reyes Pineda, H., \& Hérnandez, C. (2015). Resumen El avance investigativo a nivel ambiental ha venido creciendo en el país de manera considerable ; SOFIA - SOPHIA, 169-184. https://doi.org/ISSN (electrónico): 2346-0806

[10]. Sureda, J., Sánchez, F., \& Benayas, J. (2017). Sostenibilidad de las universidades y objetivos de desarrollo sostenible de Naciones Unidas. Universidad.

[11]. Vacio Fraga, C. (2017). Análisis de la cultura ambiental en el sector educativo del municipio de la Paz, Baja California Sur: Implicaciones y recomendaciones para el desarrollo sustentable de los recursos naturales. CIB, Centro de Investigación Biológicas del Noreste S.C.\} 